\title{
Toward Self-Managed Networks?
}

Previous successes encountered by self-* techniques (self-organization, self-healing, etc.) in biology, distributed artificial intelligence or robotics, to name only a few, have intrigued many researchers in a variety of fields. Some people are now convinced that these techniques may also be the answer to the increasingly difficult problem of designing and managing today's networks. The complexity of these networks stems from their large scale, the frequent hardware and software changes that they undergo, the high heterogeneity of their components, the complex dependencies between these components, the variety of services offered to end-users, the short time-to-market of new services and technologies, the resulting lack of debugging, etc. Can this complexity be reduced by leveraging self-management? Does the industry believe in the future of self-management in general, and self-managed networks in particular?

\section{Panelists:}

- David Bartlett, IBM Corporation, USA

- Bruno Klauser, Cisco, Switzerland

- Huw Oliver, Ericsson, Ireland

- Fabrice Saffre, BT, UK

\section{Panel Chair:}

Jean-Philippe Martin-Flatin, University of Quebec in Montreal, Canada 